\title{
White light generation using photonic crystal fiber with sub-micron circular lattice
}

\author{
Hamed Saghaei, ${ }^{*}$ Ashkan Ghanbari ${ }^{* *}$
}

\begin{abstract}
In this paper, we study a photonic crystal fiber (PCF) with circular lattice and engineer linear and nonlinear parameters by varying the diameter of air-holes. It helps us obtain low and high zero dispersion wavelengths in the visible and nearinfrared regions. We numerically demonstrate that by launching $100 \mathrm{fs}$ input pulses of 1,2 , and $5 \mathrm{~kW}$ peak powers with center wavelength of $532 \mathrm{~nm}$ from an unamplified Ti:sapphire laser into a $100 \mathrm{~mm}$ length of the engineered PCF, supercontinua as wide as 290, 440 and $830 \mathrm{~nm}$ can be obtained, respectively. The spectral broadening is due to the combined action of self-phase modulation, stimulated Raman scattering and parametric four-wave-mixing generation of the pump pulses. The third and the widest spectrum covers the entire visible range and a part of near infrared region making it a suitable source for both white light applications and optical coherence tomography to measure retinal oxygen metabolic response to systemic oxygenation.
\end{abstract}

K e y w or d s: photonic crystal fiber (PCF), white light, supercontinuum generation (SCG), dispersion engineering

\section{Introduction}

Due to the numerous applications of photonic crystal fibers (PCFs) in communication fields, they have been studied and used as nonlinear media for all optical devices. The arrangement of air holes in the cladding region gained more importance due to optical properties of PCFs such as highly nonlinearity, large and small mode areas, high numerical aperture, and adjustable zero dispersion wavelengths (ZDWs) [1-3]. To make the effective refractive index and consequently the propagation characteristics of PCF appropriate for different cases, the cladding's microstructure can be accordingly changed. This change in microstructure can be attained by making variations into the size of the physical structure. The changes in the air hole diameter $(d)$, lattice constant $\Lambda$, and lattice structure of PCFs in circular, hexagonal and square arrangements help engineers design physical structure to get important optical properties, such as dispersion, effective area and nonlinearity as desired.

Hilligse, et al demonstrated it is possible to design highly nonlinear PCFs with two ZDWs which is important specially in nonlinear applications particularly for supercontinuum generation (SCG) [4]. Ferrando, et al showed PCFs with a small core diameter can cause the zero dispersion wavelengths to be shifted to wavelengths significantly shorter than the ZDW of conventional fibers $[5,6]$. They illustrated although silica has low nonlinear refractive index but it can be compensated by designing it with a small effective area. This method results in higher nonlinear response to low pump energy. These particular properties have made PCF as an ideal candidate for nonlinear applications like SCG [7-11]. Broad- band light sources based on SCG have already found applications in various fields such as, optical communication based on dense wavelength division multiplexing [12-14], fluorescence microscopy [15], designing tunable ultrafast femtosecond laser sources [16], precise measurement of optical frequencies [17], mid-infrared SCG for spectroscopy $[9,18]$, and non-invasive imaging of sensitive surfaces based on optical coherence tomography (OCT) $[19,20]$ that uses light waves to generate cross-section images of the retina and also the light-sensitive tissue lining the back of the eye [21]. Champert, et al . demonstrated the simultaneous excitation of PCF in its normal and anomalous dispersion regimes using the fundamental and second harmonic signals of a laser that leads to a homogeneous supercontinuum (SC) in the visible and near-infrared range [22]. Kudlinski, et al. have experimentally demonstrated a SCG for OCT with a spectrum covering the region from $470 \mathrm{~nm}$ to more than $1750 \mathrm{~nm}$ by using a 300-m-long GeO2-doped-core PCF with decreasing ZDW along its length. However, the presence of $\mathrm{GeO} 2$ in silica enhances the Kerr and Raman responses of the material which is important for SCG, but the fabrication of this fiber is challenging for PCF manufacturers [23]. The SCG of low-energy ultrashort optical pulses in highly nonlinear circular silica core PCF has not been studied so far. In this paper, we present a circular silica core PCF and engineer both the dispersion and nonlinear parameters by varying the diameter of air holes and lattice constant to get the ZDW of $520 \mathrm{~nm}$. In this numerical study, we have considered the generalized nonlinear Schrödinger equation (GNLSE) for modeling the optical pulse propagation inside the fiber and to solve this equation, split step Fourier method (SSFM) has been used.

\footnotetext{
*Department of Electrical Engineering, Shahrekord Branch, Islamic Azad University, Shahrekord, Iran, h.saghaei@iaushk.ac.ir,

**Department of Electrical Engineering, Faculty of Engineering, Central Tehran Branch (CTB), Islamic Azad, University (IAU), Tehran, Iran, ashkan.ghanbari@iauctb.ac.ir
} 

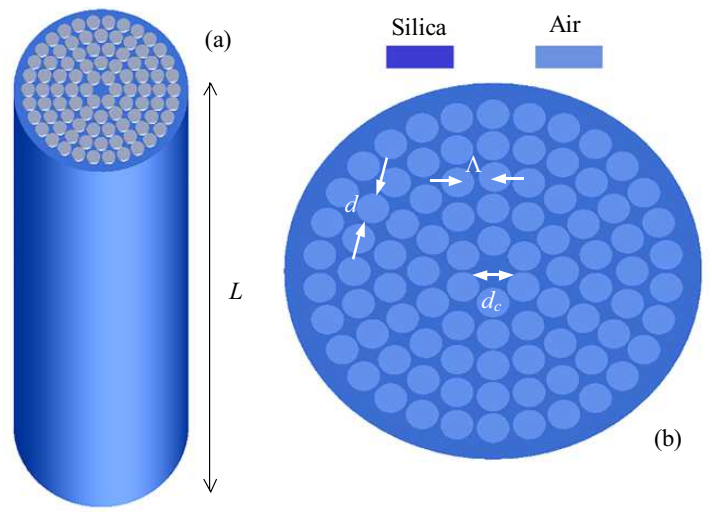

Fig. 1. (a) - Perspective view and (b) - cross-sectional view of the proposed solid core PCF , consisting of a five - ring circular lattice with submicron diameter air-holes

Our simulation results demonstrate that by launching an optical pulse with $100 \mathrm{fs}$ width, $5 \mathrm{~kW}$ peak power and center wavelength of $532 \mathrm{~nm}$ in anomalous dispersion regime into a $100 \mathrm{~mm}$ length of the PCF, a SC as wide as $830 \mathrm{~nm}$ is obtained. This spectrum covers the entire visible light and a part of infrared region that is a suitable source for both white light and optical coherence tomography applications. The details of the two fabrication techniques for PCFs can be found in [24-26] known as stack-and-draw and in [27] known as casting.

Profiles for wavelength dependence of the linear and nonlinear parameters for some diameters of air holes are depicted and a suitable one is selected. Using the calculated parameters of our desired PCF, GNLSE is rewritten and employing the numerical SSFM method is solved. Also, the spectral distributions of the PCF outputs versus the center wavelengths of the input optical pulses in the normal and anomalous dispersion regimes for different peak powers are illustrated and compared.

\section{Fiber structures and parameters}

\subsection{Structures}

We consider a solid core PCF, consisting of a fivering circular lattice with submicron diameter of air-holes in a silica as background material. The perspective and cross sectional views of the proposed PCF are shown in Fig. 1. The PCF lattice constant and length are taken to be $=700 \mathrm{~nm}$ and $L=100 \mathrm{~mm}$, respectively. In our calculations, we numerically study four PCFs with airholes diameters of $d=400,480,560$, and $660 \mathrm{~nm}$. It has already been shown that in such PCFs five air-hole rings, is large enough to make, hence the change in the PCFs dispersion is negligible when an extra ring on the outer side of the cladding is added [2]. Presence of the air holes in the cladding of a solid core PCF, whose core is basically made of the same material, makes the cladding effective refractive index smaller than that of the core. Therefore, the guiding mechanism is provided by the total internal reflection (TIR) along the solid core. It means that every optical pulse can propagate inside the PCF and the only material loss decreases its intensity which is negligible for short length of the fiber.

\subsection{Linear pameters}

In this paper, Silica is used as a background material. Its refractive index as a function of wavelength in the range of $0.21 \mu \mathrm{m} \leq \lambda \leq 3.7 \mu \mathrm{m}$ is given by four-term Sellmeier equation

$$
\begin{aligned}
& n(\lambda)=\left\{1+0.69 \lambda^{2}\left(\lambda^{2}-0.004\right)^{-1}+0.407 \lambda^{2} \times\right. \\
& \left.\times\left(\lambda^{2}-0.013\right)^{-1}+0.89 \lambda^{2}\left(\lambda^{2}-97.8\right)^{-1}\right\}^{0.5}
\end{aligned}
$$

This equation indicates that the refractive index is a decreasing function of wavelength. By solving the Maxwell equation, one can relate the dispersion parameter, $D$, to the group velocity dispersion (GVD), $\beta_{2}$, as

$$
D(\lambda)=-\frac{\lambda}{c} \frac{d^{2}}{d \lambda^{2}} \operatorname{Re}\left[n_{\mathrm{eff}}(\lambda)\right]=-\frac{2 \pi c}{\lambda^{2}} \beta_{2}(\lambda)
$$

where $c$ and $n_{\text {eff }}(\lambda)$ are the light velocity in free space and fibers effective index, respectively. In order to calculate $n_{\mathrm{eff}}(\lambda)$ for PCF, we employ a full-vector modal solver based on the finite difference time domain (FDTD) numerical method. Then using (2), dispersion profile as a function of wavelength can be calculated. Moreover, we assume anisotropic perfectly matched layers (absorbing boundaries) to be positioned outside the outer-most ring of the air-holes.

Figure 2 illustrates the dispersion profiles of PCF in terms of the input signal wavelength for four air-hole diameters of 400, 480, 560, and $660 \mathrm{~nm}$. PCF with air-hole diameter of $660 \mathrm{~nm}$ has core diameter of $d_{c}=2 \Lambda-d=740$ $\mathrm{nm}$ and the zero dispersion wavelength (ZDW) of $520 \mathrm{~nm}$ that are less than those in other PCFs. Having ZDW in visible wavelength range, $520 \mathrm{~nm}$, is a key parameter for white-light SC generation. The first and second ZDWs of PCF with air-hole diameter of $660 \mathrm{~nm}$ are 520 and 890 $\mathrm{nm}$, respectively. Also, its dispersion slope and values in anomalous region (wavelengths between first and second ZDWs) are less than those in normal cases. Moreover, in anomalous region, the dispersion becomes relatively flat. The 2D schematic view of fundamental mode distribution is depicted in the inset of Fig. 2.

\subsection{Nonlinear Parameters}

Now, we investigate the nonlinear parameter of the fiber defined as

$$
\gamma=\frac{2 \pi n_{2}}{\lambda_{0} A_{\mathrm{eff}}(\lambda)},
$$

where $\lambda_{0}$ is the center wavelength, $n_{2}$ is nonlinear Kerr index of the fiber background material that is $3 \times 10^{-20}$ 


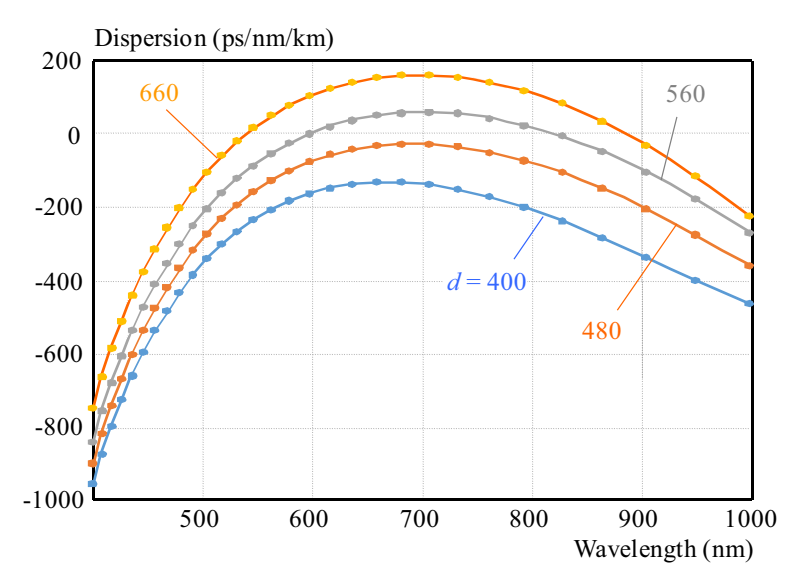

Fig. 2. Dispersion versus wavelength of PCFS of the same lattice constant, $\Lambda=700 \mathrm{~nm}$, and different air holes diameters. The inset illustrates a $2 \mathrm{D}$ schematic view of the fundamental mode distribution

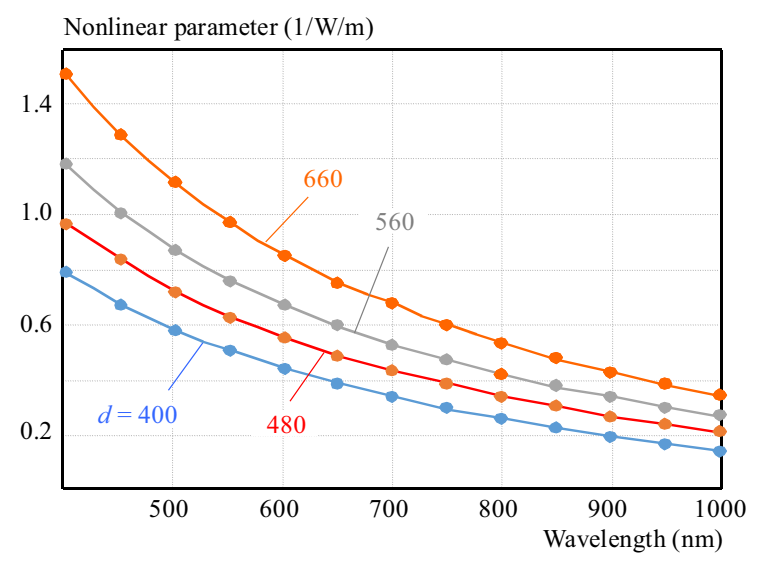

Fig. 4. Nonlinear parameter versus wavelength for PCF with lattice constant of $\Lambda=700 \mathrm{~nm}$ and different air hole diameters

$\mathrm{m}^{2} / \mathrm{W}[28]$ and $A_{\text {eff }}(\lambda)$ represents the wavelength dependence of the PCF effective cross sectional area defined as $[28]$,

$$
A_{\mathrm{eff}}(\lambda)=\frac{\left(\iint_{-\infty}^{\infty}|F(x, y)|^{2} \mathrm{~d} x \mathrm{~d} y\right)^{2}}{\iint_{-\infty}^{\infty}|F(x, y)|^{4} \mathrm{~d} x \mathrm{~d} y},
$$

where $F(x, y)$ is the optical field distribution across the $\mathrm{PCF}$ cross-section. The numerical values of the effective area calculated for PCF with different air holes diameters are depicted in Fig. 3. The PCFs nonlinear parameters versus wavelength are computed using (4) and demonstrated in Fig. 4. As can be observed from this figure, the nonlinear parameter of PCF decreases as the wavelength increases. The nonlinear parameters for a PCF with airhole diameter of $660 \mathrm{~nm}$ is higher than those for PCFs with smaller air-holes diameters, at the same wavelength.

\subsection{Generalized nonlinear Schrödinger equation}

Evolution of an optical pulse, with a slowly varying electric field amplitude represented by the envelope function $\mathrm{A}(\mathrm{z}, \mathrm{t})$, propagating along the fibers shown in Fig. 1

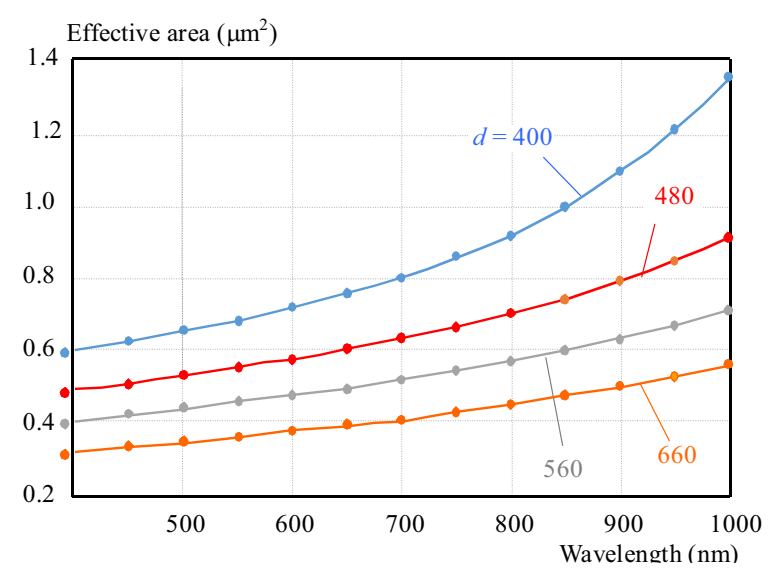

Fig. 3. Effective area versus wavelength for PCF with lattice constant of $\Lambda=700 \mathrm{~nm}$ and different air hole diameters

can be described by the solution to the generalized nonlinear Schrödinger equation (GNLSE) [28]

$$
\begin{aligned}
& \frac{\partial A}{\partial z}+\frac{\alpha}{2} A+\sum_{q=1}^{10} i^{q-1} \frac{\beta_{q}}{q !} \frac{\partial^{q} A}{\partial t^{q}}=i \gamma\left(1+\frac{i}{\omega_{0}} \frac{\partial}{\partial t}\right) \times \\
& \times A(z, t) \int_{-\infty}^{\infty} R\left(t^{\prime}\right)\left|A\left(z, t-t^{\prime}\right)\right|^{2} \mathrm{~d} t^{\prime},
\end{aligned}
$$

where $z$ and $t$ are the spatial coordinate along the fiber and the time variable, $\alpha$ is the total fiber loss, $\beta_{q}$ represents the $q$-th order dispersion parameter, $\gamma$ is the nonlinear parameter [29]. $\mathrm{R}(\mathrm{t})$ in the integrand on the right hand side of the equation is the response function including the Raman and Kerr nonlinearities. The higher order dispersions become important when the input pulse center wavelength approaches the ZDW that are given by

$$
\beta_{m}=\left.\frac{d^{m} \beta}{d \omega^{m}}\right|_{\omega=\omega_{0}},
$$

where, $\omega_{0}$ is the light center frequency. Fig. 5 shows dispersion from second to tenth orders for the desired $\mathrm{PCF}$ with air hole diameter of $660 \mathrm{~nm}$ and lattice constant of $700 \mathrm{~nm}$.

\section{Numerical simulations and discussion}

In this section, by extracting both linear parameter including dispersion orders shown in Fig. 5 and presented in Tab. 1, and also nonlinear parameter in our desired wavelength of Fig. 4, the GNLSE is rewritten for presented PCF depicted in Fig. 1. Then using SSFM, the GNLSE will be solved in both time and frequency domains for femtosecond input pulses. Several lasers emitting femtosecond pulses between $1100 \mathrm{~nm}$ and $1600 \mathrm{~nm}$ have been developed in the recent years, such as the Cr: Forsterite laser (1100 $1350 \mathrm{~nm})$ or the Er: Fibre laser $(1550 \mathrm{~nm})$ and by interacting photons with the same frequency with a nonlinear material new photons with twice 
Table 1. Values of higher order dispersions, $\beta_{n}(2 \leq n \leq 8)$, for the proposed PCF at six given center wavelengths

\begin{tabular}{ccccccc}
\hline & \multicolumn{5}{c}{ Center wavelength: $\lambda_{0}(\mathrm{~nm})$} \\
& 460 & 532 & 660 & 760 & 870 & 1064 \\
\hline$\beta_{2}$ & 31.5 & 5.53 & 35.5 & -45.4 & 19 & 114 \\
$\beta_{3}$ & $5.51 \times 10^{-2}$ & $5.78 \times 10^{-2}$ & $4.38 \times 10^{-2}$ & $2.96 \times 10^{-3}$ & $-2.09 \times 10^{-1}$ & $-8.04 \times 10^{-1}$ \\
$\beta_{4}$ & $-2.60 \times 10^{-5}$ & $1.60 \times 10^{-5}$ & $4.22 \times 10^{-5}$ & $2.62 \times 10^{-4}$ & $1.18 \times 10^{-3}$ & $3.21 \times 10^{-3}$ \\
$\beta_{5}$ & $-3.60 \times 10^{-8}$ & $-3.02 \times 10^{-8}$ & $-2.31 \times 10^{-7}$ & $-1.30 \times 10^{-6}$ & $-4.54 \times 10^{-6}$ & $-1.02 \times 10^{-6}$ \\
$\beta_{6}$ & $2.32 \times 10^{-10}$ & $-1.92 \times 10^{-10}$ & $1.63 \times 10^{-9}$ & $5.70 \times 10^{-8}$ & $1.40 \times 10^{-8}$ & $2.62 \times 10^{-8}$ \\
$\beta_{7}$ & $4.90 \times 10^{-13}$ & $-1.62 \times 10^{-13}$ & $-7.80 \times 10^{-12}$ & $-1.70 \times 10^{-11}$ & $-3.31 \times 10^{-11}$ & $-5.29 \times 10^{-11}$ \\
$\beta_{8}$ & $-2.40 \times 10^{-13}$ & $6.02 \times 10^{-13}$ & $2.22 \times 10^{-12}$ & $3.70 \times 10^{-12}$ & $5.75 \times 10^{-12}$ & $8.11 \times 10^{-12}$ \\
\hline
\end{tabular}
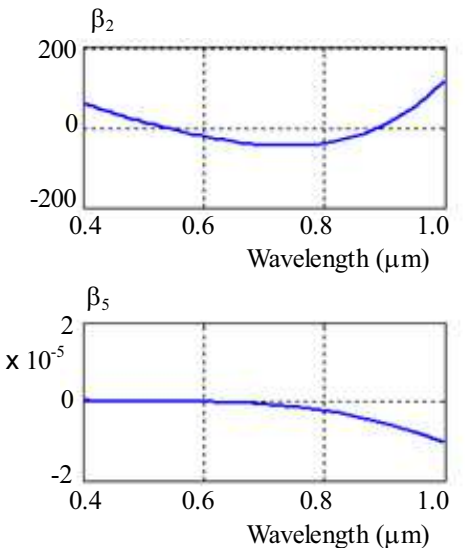

$\beta_{8}$

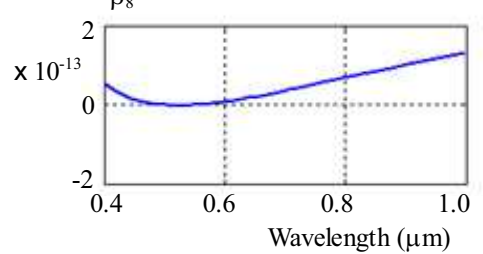

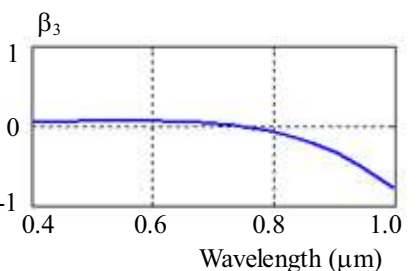

$\beta_{6}$

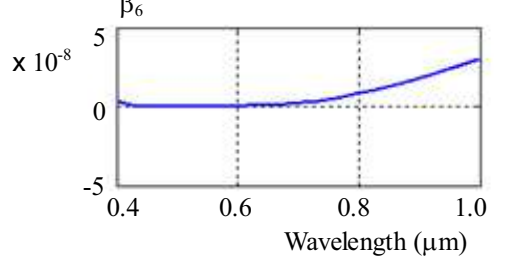

$\beta_{9}$

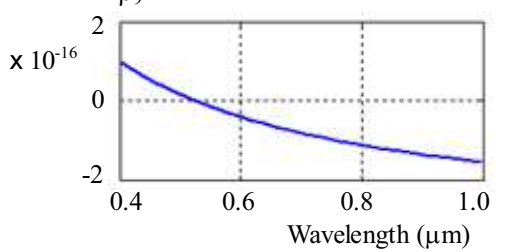

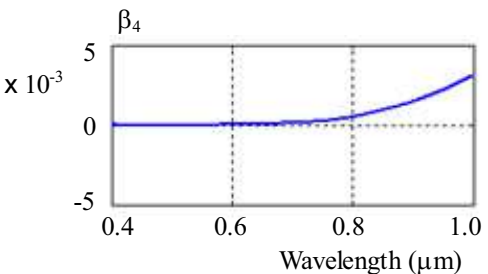

$0.4 \quad \beta_{7}$
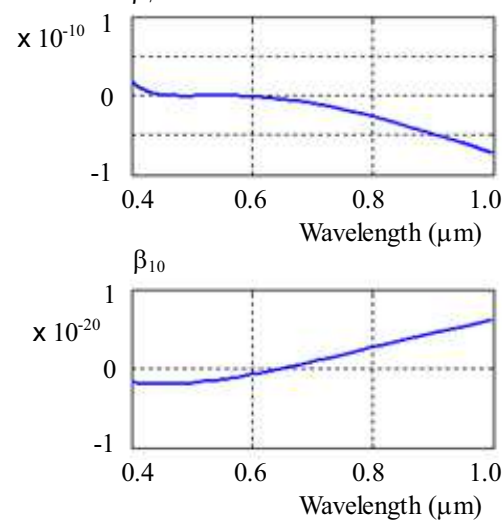

Fig. 5. Dispersion orders from $\beta_{2}$ to $\beta_{10}$ versus wavelength, for the proposed PCF with air hole diameter of $660 \mathrm{~nm}$ and lattice constant of $700 \mathrm{~nm}$

the frequency and half the wavelength of the initial photons will be achieved. Using this process a femtosecond laser source at wavelengths between 500 and 760 will be obtained.

Evolution of the spectral distribution of 100 fs optical pulse with $2 \mathrm{~kW}$ peak power, and center wavelengths of $460,532,660,760,870$, and $1064 \mathrm{~nm}$, along $100 \mathrm{~mm}$ PCF are depicted in Fig. 6. Nd:YAG lasers emitting light at $1064 \mathrm{~nm}$ with $100 \mathrm{fs}$ duration have been used in ophthalmology to correct posterior capsular opacification, a condition that may occur after cataract surgery, and for peripheral iridotomy in patients with acute angle-closure glaucoma, where it has superseded surgical iridectomy. Frequency-doubled Nd:YAG lasers (wavelength $532 \mathrm{~nm}$ ) are used for pan-retinal photocoagulation in patients with diabetic retinopathy. In certain cases, these lasers are also used to treat eye floaters. Fig. 6 shows for input optical pulses with wavelengths a bit larger than the first ZDW and less than the second one in anomalous dispersion regime, the generated supercontinua are relatively broader than other adjacent wavelengths due to the pres- ence of higher-order dispersion (HOD) and low GVD. The given results in Fig. 6 also illustrate that for central wavelengths of 532 and $870 \mathrm{~nm}$, SC spectra as wide as 440 and $450 \mathrm{~nm}$ in visible and near infrared regions have been achieved, respectively.

As can be seen in Figs. 6(a) and 6(f), the input optical pulse centered at 460 and $1064 \mathrm{~nm}$ can generate 120 and $220 \mathrm{~nm}$ spectral broadening at the end of PCF, respectively. In this case by pumping optical pulses in the normal dispersion regime, the pulses initially undergo selfphase modulation (SPM) and HOD. This causes a significant part of the light to be blueshifted. However, the minimum wavelength of the blueshift is limited by the loss that is maximized at $350 \mathrm{~nm}$. If we consider the central wavelength in the anomalous dispersion regime, the effect of SPM and soliton self-frequency shifting (SSFS) due to the stimulated Raman scattering (SRS) are predominant and responsible for broadband generated spectra. In this regime, the input pulses transform into a higher-order solitons that a small perturbation, such as HOD, SS and 

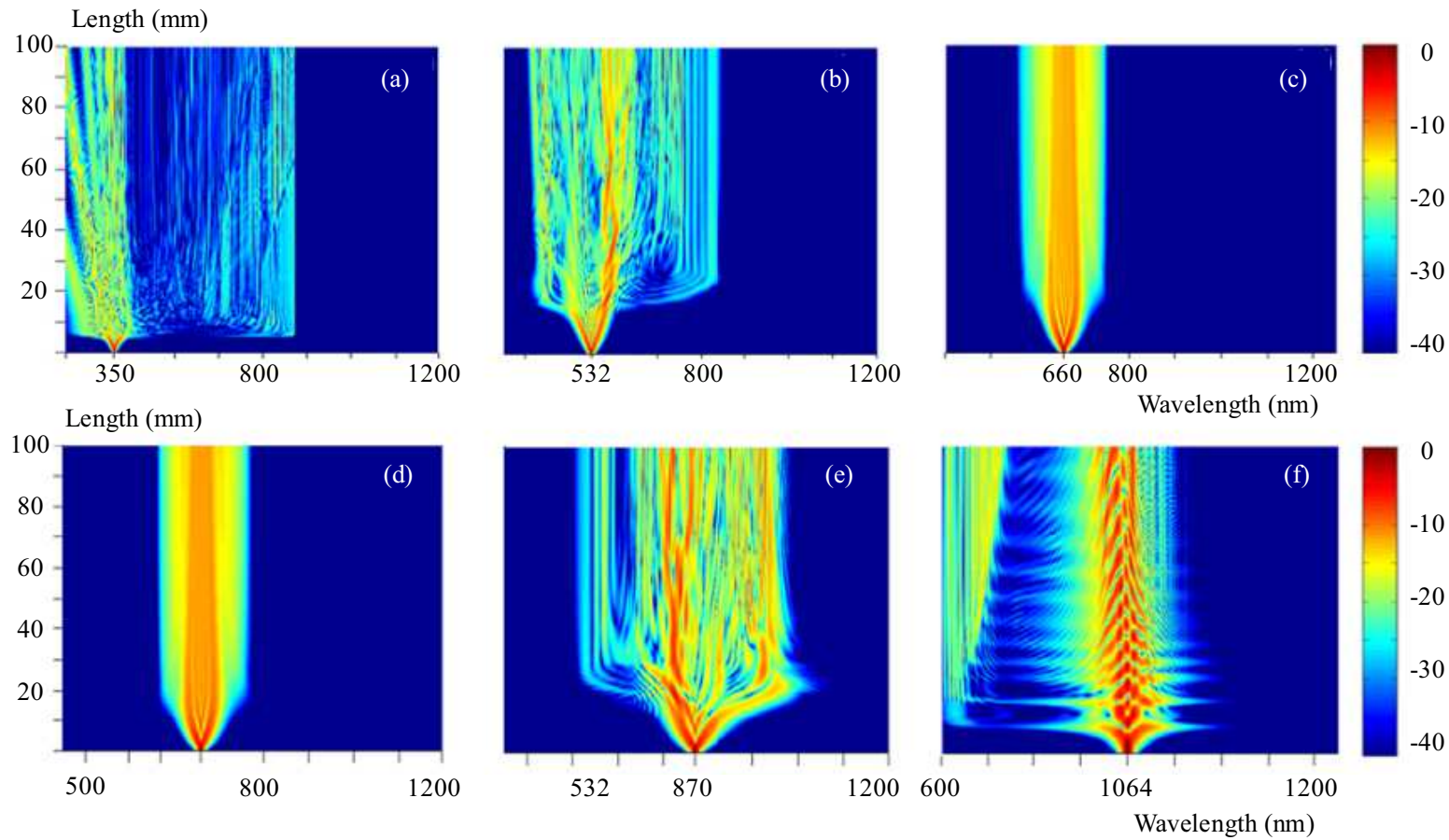

Fig. 6. Evolution of the spectral distribution of $100 \mathrm{fs}$ optical pulses of $2 \mathrm{~kW}$ peak power and central wavelengths of (a) - 460, (b) - 532, (c) $-660,(\mathrm{~d})-760,(\mathrm{e})-870$, and (f) $-1064 \mathrm{~nm}$; along a $100 \mathrm{~mm}$ PCF
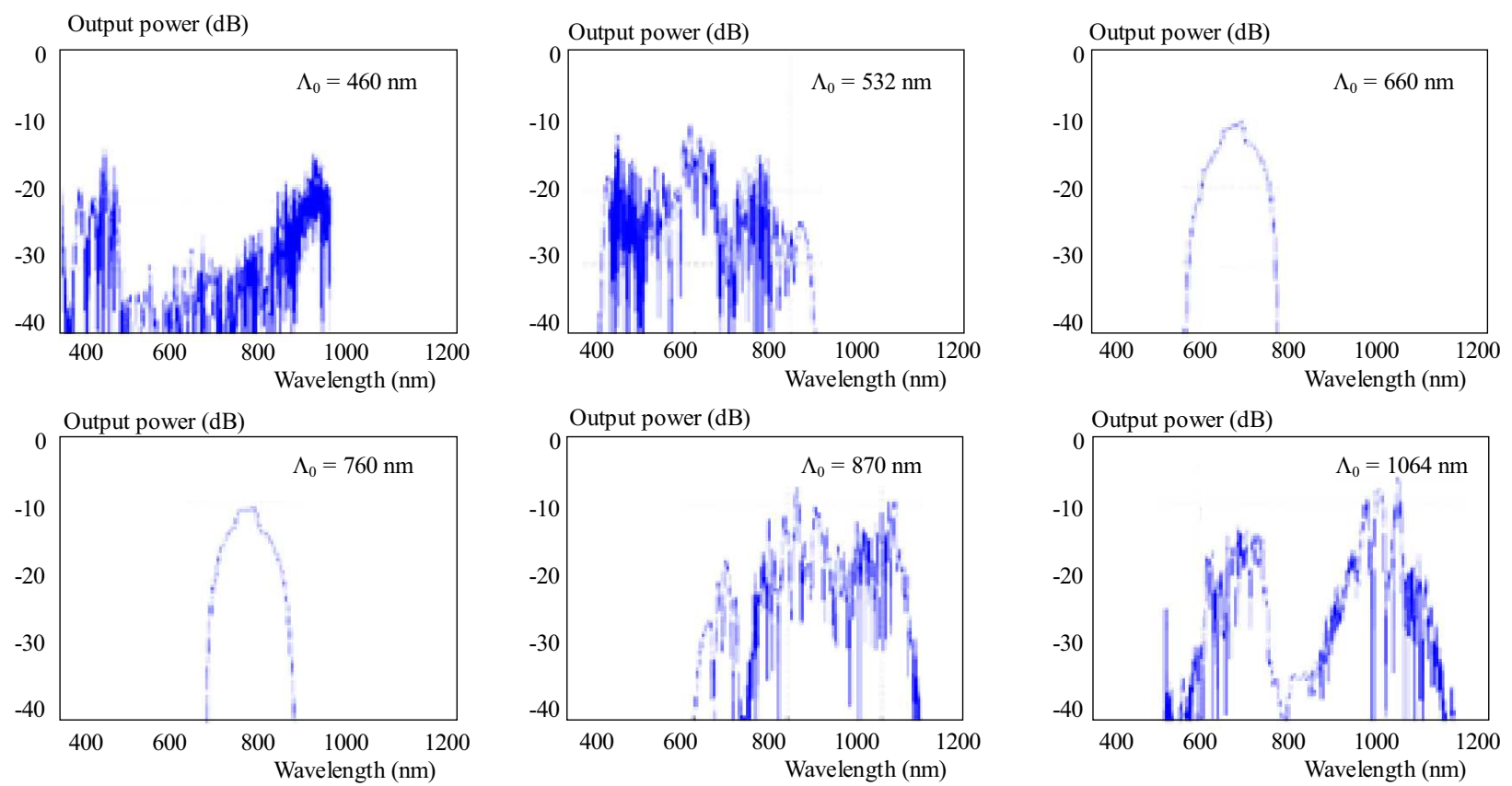

Fig. 7. Output power as a function of wavelength for $100 \mathrm{fs}$ optical pulses of $2 \mathrm{~kW}$ peak power and central wavelengths of (a) - 460 , (b) $-532,(\mathrm{c})-660,(\mathrm{~d})-760,(\mathrm{e})-870$, and (f) $-1064 \mathrm{~nm}$; in $100 \mathrm{~mm}$ length of proposed PCF

SRS can break up a given soliton of order $N$ into $N$ fundamental solitons [28], [30].

As a result, SSFS shift the wavelengths of the resulting fundamental solitons continuously toward the longer wavelengths of the spectrum, causing a considerable spectral expansion on the red side. On the other hand, nonsolitonic radiations result in broadening on the blue side of the spectrum [31]. However, both edges of blueshift and redshift are limited by the loss peak. These nonlinear processes are dominant, in a PCF of length $L$, when the nonlinear length $\left(L_{\mathrm{NL}}\right.$ and the dispersion lengths $\left(L_{\mathrm{D}}\right.$ satisfy the conditions $\left(L_{\mathrm{NL}}<L_{\mathrm{D}}\right)$ and $L_{\mathrm{NL}}<L$ [32]. Figures 6(b) - 6(e) show under such conditions, self-phase modulation leads to a symmetric spectral broadening of the optical pulse, at first. Then, the requirement for phase matching conditions are fulfilled, and hence soliton fission is occurred. Finally, self-frequency shift and non-solitonic radiations processes broaden the spectral components on both sides of the spectrum, because of which a SC is generated. For input optical pulse with central wavelength of 

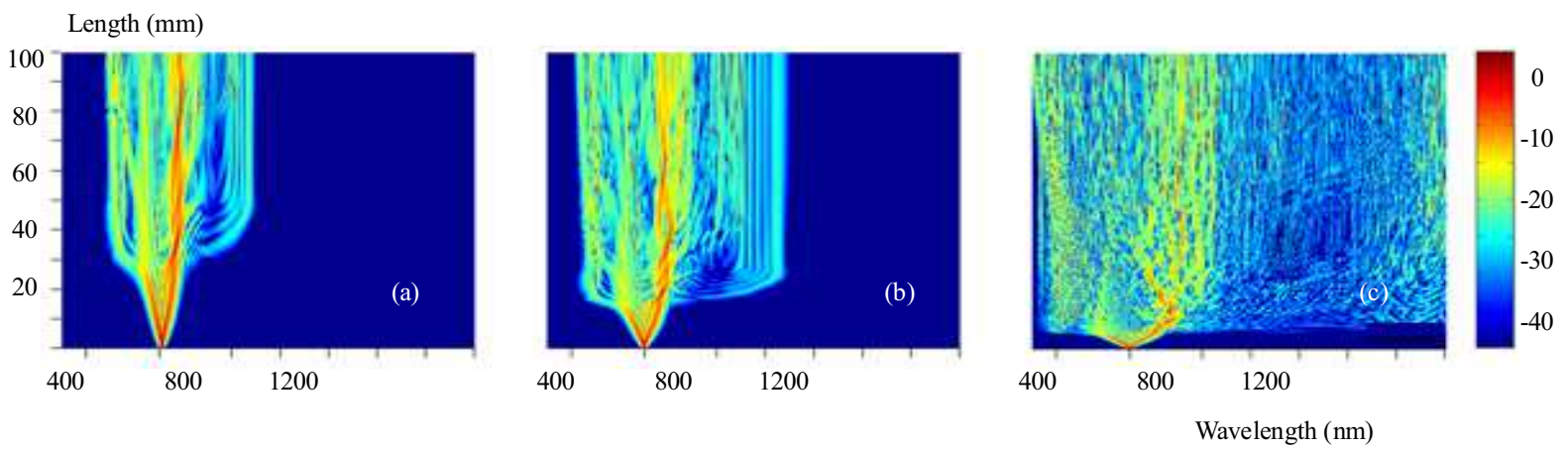

Fig. 8. Evolution of the spectral distribution of $100 \mathrm{fs}$ optical pulses with a central wavelength of $532 \mathrm{~nm}$ and peak powers of: (a) - 1 , (b) -2 , and (c) $-5 \mathrm{~kW}$; along a $100 \mathrm{~mm} \mathrm{PCF}$
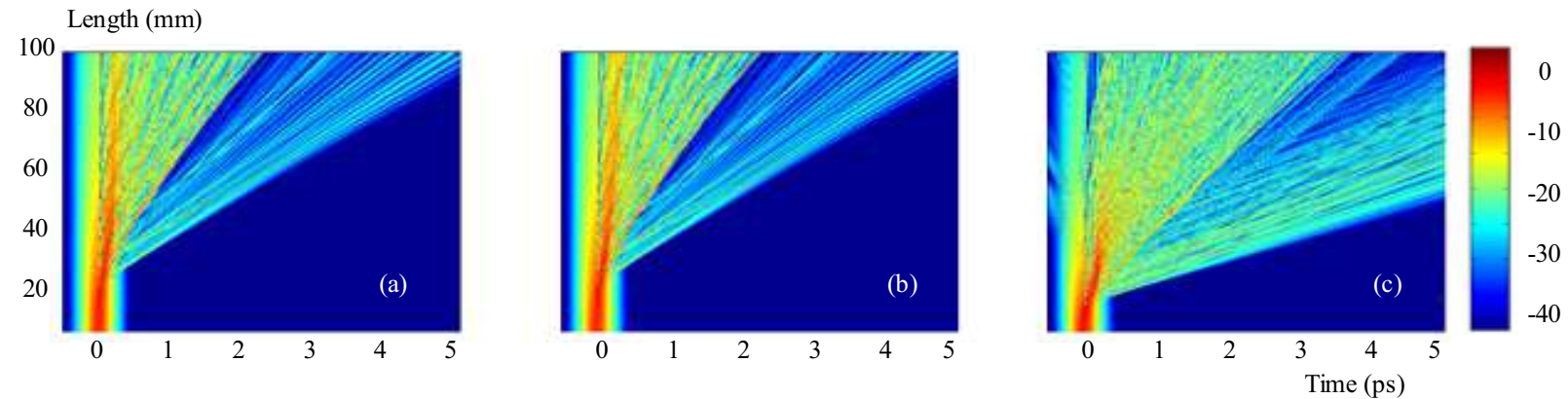

Fig. 9. Time - domain evolution of $100 \mathrm{fs}$ optical pulses with the central wavelength of $532 \mathrm{~nm}$ and peak powers of (a) - 1, (b) - 2, and (c) $-5 \mathrm{~kW}$; along a $100 \mathrm{~mm} \mathrm{PCF}$

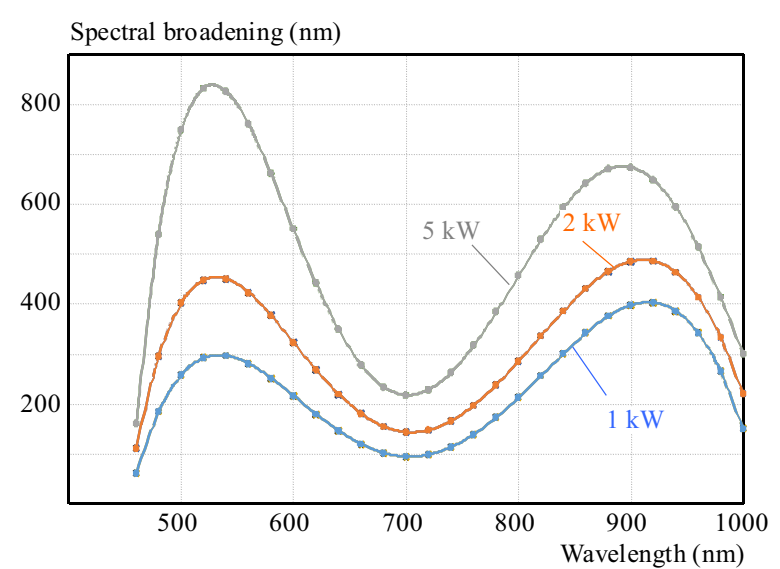

Fig. 10. Spectral bandwidth of PCF versus wavelength of input pulses for 1,2 and $5 \mathrm{~kW}$ peak powers in $100 \mathrm{~mm}$ PCF length, measured at $-30 \mathrm{~dB}$ level

$532 \mathrm{~nm}$, the second order of dispersion, $\beta_{2}$, is negative with a very small value, and $\beta_{3}$ and $\beta_{4}$ have positive and negative values, respectively. Also, for $\lambda_{0}=870 \mathrm{~nm}$, $\beta_{2}$ is negative with a very small value and $\beta_{3}$ and $\beta_{4}$ have negative and positive values, respectively. All these will help fibers to generate SC spectra in the wavelengths that we mentioned before. For $\lambda_{0}=660$ and $760 \mathrm{~nm}$, $\beta_{2}$ is negative with large value and also $\beta_{3}$ and $\beta_{4}$ have positive values.

Figure 7 shows the output power as a function of wavelength for $100 \mathrm{fs}$ input optical pulse of $2 \mathrm{~kW}$ peak power and central wavelengths of (a) -460 , (b) - 532, (c) - 660, (d) $-760,(\mathrm{e})-870$, and (f) $-1064 \mathrm{~nm}$; in $100 \mathrm{~mm}$ length of the proposed PCF. It demonstrates that flat SC spectra are achieved with a smaller bandwidth for central wavelengths of $660 \mathrm{~nm}$ and $760 \mathrm{~nm}$. In conclusion, to have SC as wide as possible, its necessary to choose central wavelength close to ZDWs in anomalous dispersion regime. However, choosing central wavelength near the first ZDW in anomalous dispersion regime generates broader SC because of its higher nonlinear parameter as shown in Fig. 4 but it has some ripples in the output power spectrum.

Figure 7 shows the output power as a function of wavelength for 100 fs input optical pulse of $2 \mathrm{~kW}$ peak power and central wavelengths of (a) 460, (b) 532, (c) 660, (d) 760, (e) 870, and (f) $1064 \mathrm{~nm}$; in $100 \mathrm{~mm}$ length of the proposed PCF. It demonstrates that flat SC spectra are achieved with a smaller bandwidth for central wavelengths of $660 \mathrm{~nm}$ and $760 \mathrm{~nm}$. In conclusion, to have SC as wide as possible, it is necessary to choose central wavelength close to ZDWs in anomalous dispersion regime. However, choosing central wavelength near the first ZDW in anomalous dispersion regime generates broader SC because of its higher nonlinear parameter as shown in Fig. 4 but it has some ripples in the output power spectrum.

Figures 8 and 9 show the evolution of the spectral and time-domain distributions along the PCF for optical pulses with $532 \mathrm{~nm}$ wavelength and 1, 2, and $5 \mathrm{~kW}$ peak powers, along $100 \mathrm{~mm}$ PCF. It reveals that by increasing the peak power of input pulses from 1 to $5 \mathrm{~kW}$, SC as wide as $830 \mathrm{~nm}$, from $390 \mathrm{~nm}$ to $1220 \mathrm{~nm}$, can be achieved in $100 \mathrm{~mm}$ PCF length that is suitable for both whit light and OCT applications. We extend our numerical study for input pulses with different central wavelengths from 400 to $1064 \mathrm{~nm}$ and assume all pulses having the same widths of 100 fs for three different peak powers of 1,2 , 
Table 2. Bandwidths of the supercontinua generated by optical signal with six different wavelengths and three different peak powers at the end of PCF in $-30 \mathrm{~dB}$ measured level

\begin{tabular}{lccc}
\hline $\begin{array}{l}\text { Peak Power } \\
\lambda_{0}(\mathrm{~nm})\end{array}$ & $\begin{array}{c}1(\mathrm{~kW}) \\
\text { Bandwidth }\end{array}$ & $\begin{array}{c}2(\mathrm{~kW}) \\
(\mathrm{nm})\end{array}$ \\
\hline 460 & 60 & 120 & 160 \\
532 & 290 & 440 & 830 \\
660 & 120 & 170 & 260 \\
760 & 125 & 175 & 280 \\
870 & 360 & 450 & 660 \\
1064 & 150 & 220 & 300 \\
\hline
\end{tabular}
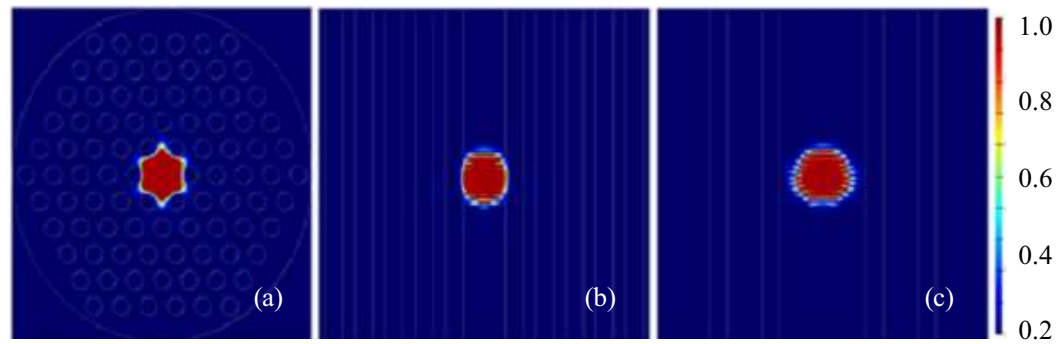

Fig. 11. Snapshots of power distributions for an input Gaussian pulse of peak power $5 \mathrm{~kW}$ and central wavelength of $532 \mathrm{~nm}$ in: (a) $x y$ ( top ) view, (b) $-x-z$ plane (side view), and (c) $-y-z$ (side view) of PCF, the scale bar on the right indicates the normalized intensity

and $5 \mathrm{~kW}$. The spectral bandwidths at the end of PCF, measured at - $30 \mathrm{~dB}$ level are shown in Fig. 10. It confirms for input optical pulses with wavelengths larger than ZDWs in anomalous dispersion regime, the generated supercontinua are relatively broader than other adjacent wavelengths caused by the presence of HOD beside low GVD. The given results in Fig. 10 also illustrate that by increasing the input pulse peak power, the spectral bandwidth at the end of PCF increases; because of the soliton fission and soliton related dynamics. The bandwidths of the supercontinua generated by the optical signals with six different wavelengths for peak powers of 1,2 and 5 $\mathrm{kW}$, through a $100 \mathrm{~mm}$ PCF, measured at - $30 \mathrm{~dB}$ level that correspond to Fig. 10, are summarized in Table 2. We have taken three snapshots of the pulse power distribution when an input Gaussian pulse of peak power $5 \mathrm{KW}$ and center wavelength of $532 \mathrm{~nm}$ is propagating along a $100 \mathrm{~mm} \mathrm{PCF}$ in $x y$ (top) and $x z$ and $y z$ (sides) cross-sectional views. These snapshots are shown in Figs. 11(a), 11(b), and 11(c), respectively. They demonstrate that the power leakage into cladding region is negligible which means PCF has a very low confinement loss due to the large diameter of air holes. The values we decided for the parameters in this simulation are close to those can be used in practice.

\section{Conclusion}

We have numerically studied and modeled supercontinuua in visible light and a part of near infrared frequency range, utilizing silica-based solid core PCF with submicron air-hole-diameter. Simulations have shown that when a 100 fs input optical pulse with the peak power of $5 \mathrm{~kW}$ and a central wavelength of $532 \mathrm{~nm}$ are used in a $100 \mathrm{~mm}$ length of PCF with air-hole diameter of $660 \mathrm{~nm}$, spectral broadening as wide as $830 \mathrm{~nm}$ in both visible and a part of near infrared regions can be obtained. Such a PCFs can be used as a suitable source for both white-light and optical coherence tomography applications.

\section{REFERENCES}

[1] A. Bjarklev, A. S. Bjarklev and J. Broeng, Photonic crystal fibres, Springer Science \& Business Media, 2003.

[2] M. Ebnali-Heidari, F. Dehghan, H. Saghaei, F. Koohi-Kamali and M. Moravvej-Farshi, "Dispersion engineering of photonic crystal fibers by means of fluidic infiltration", Journal of Modern Optics, vol. 59, no.16, pp. 1384-1390, 2012.

[3] H. Saghaei, V. Heidari, M. Ebnali-Heidari and M. R. Yazdani, "A systematic study of linear nonlinear properties of photonic crystal fibers", Optik-International Journal for Light Electron Optics, vol. 127, no.24, pp. 11938-11947, 2016.

[4] K. M. Hilligsøe, T. V. Andersen, H. N. Paulsen, C. K. Nielsen, K. Mølmer, S. Keiding, R. Kristiansen, K. P. Hansen and J. J. Larsen, "Supercontinuum generation a photonic crystal fiber with two zero dispersion wavelengths", Optics Express, vol. 12, no.6, pp. 1045-1054, 2004.

[5] A. Ferrando, E. Silvestre, J. Miret and P. Andres, "Nearly zero ultraflattened dispersion photonic crystal fibers", Optics Letters, vol. 25, no.11, pp. 790-792, 2000.

[6] A. Ferrando, E. Silvestre, P. Andres, J. Miret and M. Andres, "Designing the properties of dispersion-flattened photonic crystal fibers", Optics Express, vol. 9, no.13, pp. 687-697, 2001. 
[7] M. Ebnali-Heidari, H. Saghaei, F. Koohi-Kamali, M. Naser, M. K. Moghadasi and Moravvej-Farshi, "Proposal for supercontinuum generation by optofluidic infiltrated photonic crystal fibers", Selected Topics Quantum Electronics, IEEE Journal of, vol. 20, no.5, pp. 582-589, 2014.

[8] H. Saghaei, M. Ebnali-Heidari and M. K. Moravvej-Farshi, "Mid-infrared supercontinuum generation via As2Se3 chalcogenide photonic crystal fibers".

[9] H. Saghaei, M. Moravvej-Farshi, M. Ebnali-Heidari and M. Moghadasi, "Ultra-Wide Mid-Infrared Supercontinuum Generation $\mathrm{As}_{40} \mathrm{Se}_{60}$ Chalcogenide Fibers: Solid Core PCF versus SIF," Selected Topics Quantum Electronics, IEEE Journal of vol. 22, no.2, pp. 1-8, 2016.

[10] M. Diouf, A. B. Salem, R. Cherif, H. Saghaei and A. Wague, "Super-flat coherent supercontinuum source As 38. 8 Se 61. 2 chalcogenide photonic crystal fiber with all-normal dispersion engineering at a very low input energy", Applied Optics, vol. 56, no.2, pp. 163-169, 2017.

[11] A. Ghanbari, A. Sadr and H. T. Hesari, "Modeling photonic crystal fiber for efficient soliton-effect compression of femtosecond optical pulses at $850 \mathrm{~nm}$ ", Arabian Journal for Science Engineering, vol. 39, no.5, pp. 3917-3923, 2014.

[12] H. Saghaei, B. Seyfe, H. Bakhshi and R. Bayat, "Novel approach to adjust the step size for closed-loop power control wireless cellular code division multiple access systems under flat fading", Communications, IET, vol. 5, no.11, pp. 1469-1483, 2011.

[13] A. Mar,i, C. W. Rudy, V. G. Plotnichenko and E. M. Dianov and K. L. Vodopyanov and R. L. Byer, "Mid-infrared supercontinuum generation tapered chalcogenide fiber for producing octave-spanning frequency comb around 3m", Optics express, vol. 20, no.22, pp. 24218-24225, 2012.

[14] H. Saghaei and B. Seyfe, "New Approach to Closed-Loop Power Control Cellular CDMA Systems under Multipath Fading", , p. 1-4.

[15] Y. Sun, C. F. Booker, S. Kumari, R. N. Day, M. Davidson and A. Periasamy, "Characterization of an orange acceptor fluorescent protein for sensitized spectral fluorescence resonance energy transfer microscopy using a white-light laser", Journal of biomedical optics, vol. 14, no.5, pp. 054009-054009-11, 2009.

[16] B. W. Liu, M. L. Hu, X. H. Fang, Y. Z. Wu, Y. J. Song, L. Chai, C. Y. Wang and A. Zheltikov, "High-power wavelength-tunable photonic-crystal-fiber-based oscillator-amplifier-frequency-shifter femtosecond laser system its applications for material microprocessing", Laser Physics Letters, vol. 6, no.1, pp. 44-48, 2009.

[17] T. Udem, R. Holzwarth and T. W. Hänsch, "Optical frequency metrology", Nature, vol. 416, no.6877, pp. 233-237, 2002.

[18] H. Saghaei, M. Ebnali-Heidari and M. K. Moravvej-Farshi, "Midinfrared supercontinuum generation via As 2 Se 3 chalcogenide photonic crystal fibers", Applied optics, vol. 54, no.8, pp. 2072-2079, 2015.

[19] M. R. Avanaki, R. Cernat, P. J. Tadrous, T. Tatla, A. G. Podoleanu and S. A. Hojjatoleslami, "Spatial compounding algorithm for speckle reduction of dynamic focus OCT images", IEEE Photonics Technology Letters, vol. 25, no.15, pp. 1439-1442, 2013.

[20] M. R. Avanaki, A. Hojjat and A. G. Podoleanu, "Investigation of computer-based skin cancer detection using optical coherence tomography", Journal of Modern Optics, vol. 56, no.13, pp. 1536-1544, 2009

[21] E. A. Swanson, J. Izatt, C. Lin, J. Fujimoto, J. Schuman, M. Hee, D. Huang and C. Puliafito, "In vivo retinal imaging by optical coherence tomography", Optics letters, vol. 18, no.21, pp. 1864-1866, 1993.

[22] A. Ghanbari, A. Kashaninia, A. Sadr and H. Saghaei, "Supercontinuum generation for optical coherence tomography using magnesium fluoride photonic crystal fiber", Optik-International Journal for Light Electron Optics, vol. 140, pp. 545-554, 2017.

23] A. Kudlinski, G. Bouwmans, O. Vanvincq, Y. Quiquempois, A. Le, L. Rouge, G. Bigot, A. Mlin and Mussot, "White-light cw-pumped supercontinuum generation highly GeO 2-doped -core photonic crystal fibers", Optics letters, vol. 34, no.23, pp. 3631-3633, 2009.

24] M. Liao, C. Chaudhari, G. Qin, X. Yan, C. Kito, T. Suzuki, Y. Ohishi, M. Matsumoto and T. Misumi, "Fabrication characterization of a chalcogenide-tellurite composite microstructure fiber with high nonlinearity", Optics express, vol. 17, no.24, pp. 21608-21614, 2009.

25] C. Yi, P. Zhang, F. Chen, S. Dai, X. Wang, T. Xu and Q. Nie, "Fabrication characterization of Ge20Sb15S65 chalcogenide glass for photonic crystal fibers", Applied Physics B, vol. 116, no.3, pp. 653-658, 2014.

26] K. Morishita and Y. Miyake, "Fabrication resonance wavelengths of long-period gratings written a pure-silica photonic crystal fiber by the glass structure change", Journal of Lightwave Technology, vol. 22, no.2, pp. 625-630, 2004.

27] Q. Coulombier, L. Brill,, P. Houizot, T. Chartier, T. N. N'guyen, F. Smektala, G. Renversez and A. Monteville and D. Mchin and T. Pain, "Casting method for producing low-loss chalcogenide microstructured optical fibers", Optics Express, vol. 18, no.9, pp. 9107-9112, 2010.

[28] G. P. Agrawal, Nonlinear fiber optics Springer, 2000.

[29] C. Agger, C. Petersen, S. Dupont, H. Steffensen, J. K. Lyngs, C. L. Thomsen, J. Thgersen, S. R. Keiding and O. Bang, "Supercontinuum generation ZBLAN fibers-detailed comparison between measurement simulation", Optical Society of America. Journal B: Optical Physics, vol. 29, no.4, pp. 635-645, 2012.

30] M. Ebnali-Heidari, M. K. Moravvej-Farshi and A. Zarifkar, "Multichannel wavelength conversion using fourth-order soliton decay", Journal of Lightwave Technology, vol. 25, no.9, pp. 2571-2578, 2007.

31] A. V. Husakou and J. Herrmann, "Supercontinuum generation, four-wave mixing, fission of higher-order solitons photonic-crystal fibers", JOSA B, vol. 19, no.9, pp. 2171-2182, 2002.

$32]$ J. M. Dudley, G. Genty and S. Coen, "Supercontinuum generation photonic crystal fiber", Reviews of modern physics, vol. 78, no.4, pp. 1135, 2006.

Received 6 October 2016

Hamed Saghaei was born in Shahrekord in 1982. He received the BSc, MSc and PhD degrees from Amirkabir University of Technology, Shahed University, and Science and Research of IAU, Tehran, Iran all in electrical engineering in 2004, 2007, and 2015, respectively. Now, he is with the department of electrical engineering, Shahrekord Branch, Islamic Azad University as assistant professor. He has a good collaboration in doing research with some groups in Massachusett Institute of Technology (MIT), the University of Alberta. His research interests and studies are spread spectrum OCDMA-based communications, Photonic crystal fibers and waveguides, plasmonic, nanostructures, and solar cells.

Ashkan Ghanbari was born in Karaj, Iran in 1985. He received his BSc, MSc from Tehran Emam Hussein University and Qazvin Islamic Azad University in 2009 and 2012, respectively, all in electrical engineering. He is now a PhD student at Tehran Central Branch, Islamic Azad University (IAUTCB) in electrical engineering. His principal research areas are fiber optics, photonic crystal fibers and fiber lasers. He has published several reports in national and international journals and conference proceeding. 